\title{
Adaptation of the agyrophil technique for nucleolar organizer regions to canine peripheral blood smears
}

\author{
John Ogunsola ${ }^{1, *}$ and Richard Antia ${ }^{2}$ \\ ${ }^{1}$ Veterinary Teaching Hospital, University of Ibadan, Ibadan, Nigeria \\ ${ }^{2}$ Department of Veterinary Pathology, University of Ibadan, Ibadan, Nigeria
}

\begin{abstract}
Nucleolar organizer regions (NORs) are chromosomal segments which are selectively stained by silver methods and evaluated as agyrophilic nucleolar organizer regions (AgNORs). The evaluation of AgNORs provides an insight into the level of cellular proliferation. This technique has extensively and sparsely been used in human and veterinary histopathology respectively. However, two major drawbacks have been irreproducible results and excessive staining precipitates. This study seeks to adapt the technique to canine peripheral blood smears in order to establish a routine staining protocol. Standardized volumes and concentrations of silver nitrate, gelatin, and formic acid were applied to smears at different temperatures for varying lengths of time. The technique was applied to unfixed and fixed smears. In some cases, a reducing agent ( $1 \%$ potassium iodide) was applied. It was shown that the optimum staining protocol was achieved by applying standardized solutions to a fixed smear at $46^{\circ} \mathrm{C}$ for 50 minutes. It is concluded that the staining protocol outlined in this study is practicable, and produces excellent and reproducible results that would enhance evaluation of AgNORs in canine peripheral blood cells.
\end{abstract}

Keywords: AgNOR, Canine blood smears, Cytology, Veterinary hematology.

\section{Introduction}

The use of proliferation markers for the study of physiologic and pathologic processes has always attracted interest from researchers (Egan and Crocker, 1992). Markers that have been employed include mitotic index, Ki-67, proliferating cell nuclear antigen (PCNA) as well as the silver-staining technique of demonstrating agyrophilic nucleolar organizer regions (AgNORs) (Ceyhan et al., 1996).

The agyrophilic technique was originally developed and applied to chromosomes (Goodpasture and Bloom, 1975), modified into a single step process (Howell and Black, 1980) and initially applied to histologic sections by Ploton et al. (1986). The one-step procedure succinctly involves the use of two parts of a $50 \%$ silver nitrate and one part of $2 \%$ gelatin dissolved in $1 \%$ formic acid at $20^{\circ} \mathrm{C}$ (Ploton et al., 1986). Ever since Ploton and his colleagues introduced the technique to histologic sections, numerous researchers have applied it to various tissues and the procedure has gained ground in human pathology and haematology (Metze et al., 1999; Bukhari et al., 2007; Strefezzi et al., 2009). However, there are a few reports of its application to tissues of the dog (Jelesijevic et al., 2003; Vajdovich et al., 2004).

A sizeable body of literature has developed over time evaluating this technique in various diagnostic applications. It has not been reported that the technique was applied to peripheral blood smears of veterinary species.

Despite success with the use of the agyrophilic technique, there are also comments about problems with the staining protocol that may make it difficult in interpreting results.

Some of the problems include limited reproducibility, background staining of tissues, deposition of precipitates on the slides (Crocker et al., 1989), as well as variation in the staining intensity of Nucleolar organizer regions (NORs) (Manu et al., 2006), with respect to the fixative used, staining time, staining temperature; fading of slides in a short time (Lindner, 1993 ) etc. Various authors have advanced and identified reasons for the variation. Some of the reasons identified include dirty glassware or impure water (Crocker et al., 1989), the type of colloidal developer used and pre-reduction of the tissue (Lindner, 1993); and consequently developed their own variations of the staining protocol to eliminate these drawbacks.

These variations have included post-staining techniques to preserve the staining quality (Bukhari et al., 2007) and counter-staining with other stains (Vajdovich et al., 2004).

In our own experience, numerous factors affect the final outcome of the staining quality; and it appears that different tissue preparations would require different adaptations of this valuable technique. Therefore many established factors governing the staining procedure were experimented. 
This paper aims to develop and outline a standardized staining protocol applicable to domestic dog's (Canis familiaris) peripheral blood smears, considering time and cost, that would produce optimum staining quality when evaluating AgNORs in lymphocytes.

\section{Animals and Sampling}

\section{Materials and Methods}

Fifty blood samples (about $3 \mathrm{mls}$ each) were obtained by cephalic phlebotomy from same number of dogs presented to the Small Animal Clinic of the Veterinary Teaching Hospital, University of Ibadan. The clients were well informed about the need for blood collection and their consent was obtained. About $2.5 \mathrm{ml}$ of the blood obtained was emptied into an EDTA-containing tube. A heparinized capillary tube was filled from the remaining blood in the syringe. All blood smears were fixed immediately (see effect of fixatives), and stained with the agyrophil technique in an incubator. During the staining with agyrophil technique, variations in the reagents, temporal, and environmental factors were tested. After staining, each smear was evaluated qualitatively, blindly and independently by two pathologists using Olympus ${ }^{\circledR}$ CX-21 microscope at 1000X magnification.

\section{Effect of Anticoagulants}

Thin blood smears from each of the canine patient were made in triplicates: one each from the blood in the syringe, the heparinized capillary tube, and EDTAcontaining tube.

\section{Effect of Fixatives}

Blood smears were subjected to three different fixatives while others were air-dried and devoid of any fixative. The fixatives included 95\% methanol; 100\% ethanol; acetone as proposed by Metze et al. (1999) and Carnoy's solution (comprising absolute ethanol: glacial acetic acid 3:1 (v/v)) as proposed by Trere (2000).

\section{Effect of Pre-Reduction of slides}

It had been noted that pre-reduction of slides produced better staining quality (Lindner, 1993) and two reduction agents (Cleland's reagent and 1\% potassium iodide) were suggested. In our experience, Cleland's reagent was not readily available and hence $1 \%$ potassium iodide was used.

\section{Preparation and Filtration of Staining Solution}

Colloidal silver staining solution was prepared by dissolving gelatin in $1 \%$ aqueous formic acid at a concentration of $2 \%$ and mixing one volume of this solution (referred to as Solution A) with two volumes of $50 \%$ silver nitrate (Solution B). In some instances, Solution A and Solution B were filtered using a Whatmann ${ }^{\circledR}$ filter while in others only one of both solutions were filtered before mixing to form the final staining solution.

\section{Staining Time}

As observed by researchers in the field (Lindner, 1993), the duration of staining influences on the resolution and identification of AgNORs. The challenge was to find a suitable time of staining that would allow weak AgNORs to be identified and as well not over-stain highly reactive NORs. Staining times employed ranged from 15 minutes to about 6 hours (although other variables such as staining temperature played a role in the length of time). However, following standardized protocols and considering practicality, four different durations were compared: 15minutes, 30minutes, 1hour, and 2hours.

\section{Staining Temperature}

Four different temperatures were employed in an incubator to look for optimum staining quality as well as practicality in routine haematology in terms of staining time. The temperatures were $20^{\circ} \mathrm{C}, 37^{\circ} \mathrm{C}$, $46^{\circ} \mathrm{C}$, and $70^{\circ} \mathrm{C}$, starting with both extremes before choosing some midpoints.

\section{Effect of Anticoagulants \\ Results and Discussion}

There was no visual difference in the staining quality observed by the pathologists in smears made from samples containing EDTA, heparin and those that did not contain either EDTA or heparin.

\section{Effect of Fixatives}

The smears that were not fixed at all generally required very long periods (between 5 and 6 hours) of staining and even with such prolonged periods, the staining intensity was at best faint. Smears fixed with acetone generally had a lot of background precipitate that obscured AgNORs and made resolving AgNORs very difficult, while those fixed with the improvised Carnoy's solution presented with moderate amounts of background precipitates. Good staining results were obtained with $100 \%$ ethanol and $95 \%$ methanol. The best quality of staining cells was achieved with $95 \%$ methanol, while cells lost some degree of nuclear and cytoplasmic details when $100 \%$ ethanol was used.

\section{Effect of Pre-Reduction of slides}

Smears that were pre-reduced with $1 \%$ potassium iodide showed less background staining, distinct AgNORs and more uniform staining of the smear. Smears that were not exposed to the reducing agent showed intense staining, and background stain precipitates.

\section{Effect of Filtration of Staining Solution}

Smears that were stained with filtered Solutions A and B lacked stain precipitates. Stain precipitates were numerous in smears that were stained with a mixture of unfiltered Solutions A and B, as well as those stained with a mixture containing unfiltered Solution A.

\section{Effect of different staining times}

At room temperature, 15 and 30 minutes were insufficient to obtain well-stained smears. After 1hour, slides began to be fairly stained. At 2 hours, slides were observed to be over-stained with AgNORs appearing smudged and difficult to resolve from one another. 


\section{Effect of Staining Temperature}

At least 6 hours were required to obtain good staining at $20^{\circ} \mathrm{C}$. However, the authors considered it impracticable as a routine. Even with short staining times, it was difficult to resolve highly reactive NORs when staining was done at $70^{\circ} \mathrm{C}$. Optimum staining quality at $37^{\circ} \mathrm{C}$ required times of between $1 \frac{1}{2}$ hours and 2hours, while at $46^{\circ} \mathrm{C}$ slides could be stained in $45-50$ minutes with a good balance struck in the visualization of both highly and less reactive NORs.

\section{Outline of modified AgNOR Staining Protocol}

1. Fix smears with $95 \%$ methanol for 5 minutes. Allow to dry

2. Prepare $1 \%$ potassium iodide (reducing agent). Flood slide with $0.5 \mathrm{ml}$ for 30 minutes.

3. Dissolve $2 \%$ gelatin in $1 \%$ formic acid (A). Allow to dissolve completely. Filter.

4. Prepare fresh $50 \%$ silver nitrate (B). Filter.

5. Mix A and B in ratio 1:2. Avoid sediments.

6. Drop $0.3 \mathrm{ml}$ of the staining solution on each slide

7. Incubate at $46^{\circ} \mathrm{C}$ for 50 minutes

8 . Rinse thoroughly in flowing water or several changes of distilled water; and wipe dry with filter paper.

9. Optional: Mount with cover-slip

Outcome: NORS are stained as black/brown dots in a yellow nucleus (Fig. 1).

The reason why anti-coagulants do not have effect on the staining outcome is not known. This finding corroborates Lindner (1993), who did not find any difference in the staining quality from blood samples containing EDTA and those that did not. This finding portends good for the clinician or researcher as smears can be made at the point of collection/phlebotomy or during sample analysis in the laboratory.

Our results suggest that the use and type of fixative has a profound effect on the outcome of the agyrophil technique. Our finding that acetone produces bad staining quality disagrees with some authors (Metze et al., 1999), although they employed acetone for human blood smears. Alcohol based fixatives produce good results (Lindner, 1993). The use of sprays with aqueous forms of alcohol-based fixatives was not compared.

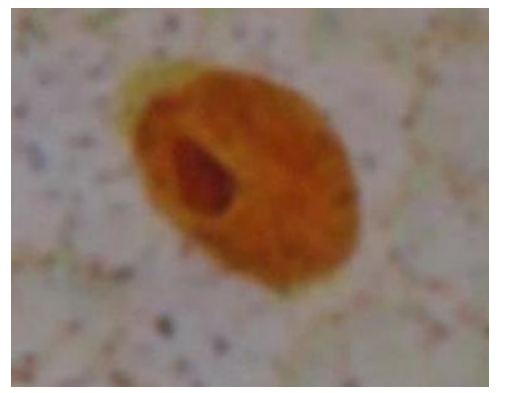

Fig. 1. Canine peripheral blood smear. Brownish agyrophilic nucleolar organizer region in the yellow nucleus of a lymphocyte. AgNOR technique (Objective 100×).
A major step that contributed to a decrease in the background staining is reduction (Lindner, 1993). Exposing the slides to $1 \%$ potassium iodide produced smears of better and much more uniform staining quality when compared with those that were not reduced. No ascertain if different reducing agents suggested by Lindner (1993) produced uniform or varying effects.

It could be beneficial to incorporate reducing agents into the fixatives, stock solution of $2 \%$ gelatin dissolved in $1 \%$ formic acid or into the final staining solution, aiming to eliminate the separate process of first reducing the slide post-fixation. The volume and concentration of the reducing agent, if the method of incorporation is to be explored, would need to be worked out.

The effect of temperature cannot be over-emphasized. It was noted that mixing Solutions $\mathrm{A}$ and $\mathrm{B}$ is an endothermic reaction and as such the rate of reaction would increase with increasing temperatures. Increasing staining temperature decreases staining time agreed with a number of authors (Ploton et al., 1986; Crocker et al., 1989; Lindner, 1993). Controlled temperature is required for reproducibility of the staining quality (Howell and Black, 1980). The choice of $46^{\circ} \mathrm{C}$ as a routine staining temperature is based on the practicable staining time that it affords. Except for the DiffQuik ${ }^{\circledR}$, which is used in 3 minutes, many stains routinely used in examining blood smears, are applied during 30 to 40 minutes.

This makes the procedure easily incorporable as routine if and when needed. Also, with the combination of the proposed staining time and temperature, it is easier to visualize NORs whether they are highly or less reactive.

In comparison to other reagents used in the process, silver nitrate is expensive. It would be desirable that protocols requiring less amount of such compound could be developed. The probable ways of achieving this would include reducing the concentration of silver nitrate employed in the process as well as limiting the volume of silver nitrate solution required to stain a smear.

It is reported that $33 \%$ silver nitrate produced good staining results (Jelesijevic et al., 2003). However, Lindner (1993), disagrees with this view as he observed that reduction of silver concentration reduced the rate of staining to a point where staining times became impracticable and therefore suggested that the concentrations originally reported by Ploton et al. (1986) were about optimum. Based on this finding, attention to reducing the volume of staining solution required per slide was giving attention. The coplin jar variant of slide positioning during staining did not provide this advantage. 
However, both the up-facing and down-facing variants required less volume (about $0.3 \mathrm{ml}$ ) per slide. A dropper can be used to pipette 3 drops on the smear.

It can be concluded that the agyrophil technique is applicable to canine peripheral blood smears, and that various factors affect the final quality of the staining of NORs. A draw-back to the outlined protocol would be the extra time and/or cost incurred from the prereduction stage. The extra time may become inconsequential as one would need to freshly prepare the silver nitrate solution. While the silver nitrate solution is being prepared, the slides can be reduced with potassium iodide. It can be suggested that the outlined protocol could be routinely employed to canine blood smears.

\section{Conflict of interest}

The authors declare that there is no conflict of interests.

\section{References}

Bukhari, M.H., Niazi, S., Khan, S.A., Hashmi, I., Perveen, S., Qureshi, S.S., Chaudhry, N.A., Qureshi, G.R. and Hasan, M. 2007. Modified method of AgNOR staining for tissue interpretation in histopathology. Int. J. Exp. Pathol. 88, 47-53.

Ceyhan, K., Akpolat, I., Kobat, I., Bozdogan, N. and Erekul, S. 1996. AgNORs, PCNA and histologic activity index in chronic liver disease. Turkish $\mathrm{J}$. Med. Sci. 29, 11-15.

Crocker, J., Boldy, D.A.R. and Egan, M.J. 1989. How should we count AgNORs? Proposals for a standardized approach. J, Pathol, 158, 185-188.

Egan, M.J. and Crocker, J. 1992. Nucleolar organizer regions in pathology. Br. J. Cancer 65, 1-7.

Goodpasture, C. and Bloom, S.E. 1975. Visualization of nucleolar organizer regions in mammalian chromosomes using silver staining. Chromosoma
53(1), 37-50.

Howell, W.M. and Black, D.A. 1980. Controlled silverstaining of nucleolus organizer regions with a protective colloidal developer: a 1-step method. Experientia 36, 1014-1015.

Jelesijevic, T., Jovanovic, M., Knezevic, M. and Aleksic-Kovacevic, S. 2003. Quantitative and qualitative analysis of AgNOR in benign and malignant canine mammary gland tumors. Acta Veterinaria 53(5), 353-360.

Lindner, L.E. 1993. Improvements in silver-staining technique for nucleolar organizer regions. $\mathrm{J}$. Histochem. Cytochem. 41(3), 439-445.

Manu, V., Rajaram, T. and Rai, R. 2006. Value of silver binding nucleolar organizer regions in squamous cell carcinomas of upper aero-digestive tract. Med. J. Armed Forces India 62, 123-128.

Metze, K., Chiari, A.C., Andrade, F.L. and LorandMetze, I. 1999. Changes in AgNOR configurations during the evolution and treatment of chronic lymphocytic leukemia. Hematol. Cell Ther. 41(5), 205-210.

Ploton, D., Menager, M., Jeannesson, P., Himber, G., Pigeon, F. and Adnet, J.J. 1986. Improvement in the staining and visualization of the agyrophilic proteins of the nucleolar organizer region at the optical level. Histochem. J. 18, 5-14.

Strefezzi, R.F., Kleeb, S.R., Xavier, J.G. and Calaodias, J.L. 2009. Prognostic indicators for mast cell tumors. Brazilian J. Vet. Pathol. 2(2),110-121.

Trere, D. 2000. AgNOR staining and quantification. Micron 31, 127-131.

Vajdovich, P., Psader, R., Toth, Z.A. and Perge, E. 2004. Use of the agyrophilic nucleolar region for cytologic and histologic examination of lymph nodes in dogs. Vet. Pathol. 41, 338-345. 